\title{
'Labyrinth', una aplicación móvil para el aprendizaje basado en problemas: evaluación de los usuarios sobre su eficacia como herramienta de aprendizaje.
}

\author{
Rocío Fortún Rabadán ${ }^{\text {a,b }}$, Lindsey Bruton ${ }^{\mathrm{c}, \mathrm{d}}$, Carolina Jiménez Sánchez ${ }^{\mathrm{a}, \mathrm{b}}$, María \\ Ortiz Lucas, ${ }^{\mathrm{a}, \mathrm{b}}$, Miriam Clavel Rodríguez .
}

aDepartamento de Fisioterapia. Universidad San Jorge. Zaragoza (rfortun@usj.es; cjimenez@usj.es; mortiz@usj.es; alu.23159@usj.es) brupo de Investigación iPhysio. Universidad San Jorge. Zaragoza. 'Instituto de Lenguas Modernas. Universidad San Jorge. Zaragoza (labruton@usj.es). dMITLHE, Multidisciplinary Innovation in Teaching and Learning. Universidad San Jorge. Zaragoza.

\begin{abstract}
Current learning theories promote the employment of active methodologies, placing the learner at the centre of the learning process as protagonist. Both problem and case-based learning, methodologies embracing this perspective, have been widely used in health sciences.

Other intrinsic factors key to learning such as self perception, motivation and confidence also play an important role in the learning process and outcomes.

This paper focuses on student perceptions of 'Labyrinth', a web-based application which permitted learners to solve cases requiring electrotherapy treatment. An anonymous online questionnaire was developed to evaluate students' perceptions of how 'Labyrinth' contributes to and supports learning. The contents of the questionnaire aimed to raise awareness in the learner of the importance of personal effort and self-motivation in achieving learning goals.

Direct and relevant correlations were found between students perceptions about the global utility of 'Labyrinth', and their self-perceived levels of confidence, motivation, effort and cognitive development during the use of the application.

These findings highlight the need for reflection on these intrinsic learning factors in order that awareness and consequent development of key learning habits are encouraged.
\end{abstract}

Keywords: Case-based learning; web-based application; intrinsic learning factors; self-awareness; self-motivation; physiotherapy; electrotherapy. 


\section{Resumen}

Las teorias actuales del aprendizaje proponen la aplicación de metodologías activas, situándose el alumno en el centro del proceso de aprendizaje. Dentro de estas metodologías encontramos el aprendizaje basado en problemas o en casos, ampliamente utilizado en Ciencias de la Salud.

Otros factores intrínsecos claves para el aprendizaje como la auto-percepción, la motivación y la confianza, juegan un papel importante en el proceso y los resultados del aprendizaje.

Este articulo se centra en las percepciones de los estudiantes sobre 'Labyrinth', una aplicación web que permite resolver casos que requieren tratamiento electroterápico.

Se desarrolló un cuestionario anónimo online para evaluar las percepciones de estudiantes de Fisioterapia respecto a cómo 'Labyrinth' contribuye y apoya el aprendizaje. El contenido del cuestionario tenía como objetivo sensibilizar sobre la importancia del esfuerzo personal y la auto-motivación para alcanzar los objetivos de aprendizaje.

Se encontraron correlaciones directas y relevantes entre las percepciones de los estudiantes acerca de la utilidad global de 'Labyrinth' y sus niveles de confianza, motivación, esfuerzo y desarrollo cognitivo autopercibidos durante su uso.

Estos hallazgos evidencian la necesidad de reflexión sobre estos factores intrinsecos de aprendizaje a fin de fomentar la concienciación y el consiguiente desarrollo de los hábitos de aprendizaje fundamentales.

Palabras clave: Aprendizaje basado en casos; aplicación online; factores intrínsecos del aprendizaje; auto-percepción; fisioterapia; electroterapia.

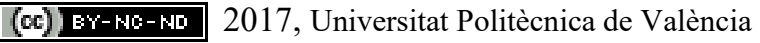




\section{INTRODUCCIÓN}

Este estudio se centra en valorar la utilidad de 'Labyrinth' para asistir y contribuir al aprendizaje en base a la percepción de los usuarios. 'Labyrinth' consiste en una aplicación online basada en la resolución de casos clínicos, diseñada para facilitar a los estudiantes de fisioterapia la consecución de los objetivos de aprendizaje, así como fomentar su reflexión sobre sus propios recursos. Con estos fines, en el desarrollo de esta herramienta innovadora y de los métodos para su evaluación se tuvieron en cuenta muchos factores que han evidenciado beneficios en el área de la pedagogía, y que se exponen a continuación.

\subsection{Factores intrínsecos y Aprendizaje basado en problemas.}

El trabajo de la psicóloga Carol S. Dweck (Dweck, 2007) sobre motivación y teorías implícitas de la inteligencia, identificó en la población de estudio dos modalidades de creencias sobre la inteligencia, a las que denominó respectivamente "inteligencia fija" presunción de que la inteligencia es una cualidad estática que se posee o no se posee-, e "inteligencia en desarrollo" -convicción de que esta cualidad es plástica y por tanto puede aumentarse-. Varias investigaciones mostraron que el tipo de creencia que los estudiantes tienen sobre la inteligencia repercute directamente en sus resultados académicos, y que educarles en la creencia de la inteligencia en desarrollo aumenta su seguridad en sus posibilidades y por tanto mejora dichos resultados de manera significativa (Blackwell, 2007; Good, 2003).

La auto-percepción de los estudiantes respecto a otros aspectos también ha mostrado influir en el rendimiento académico (Pajares,1996). La auto-percepción de la capacidad de esfuerzo se relaciona directamente con la creencia de una capacidad de aprendizaje creciente, dado que la inteligencia y el desarrollo de competencias deben acompañarse necesariamente de esfuerzo (Dweck, 2007). En base a ello, el esfuerzo garantiza la posibilidad de obtener buenas calificaciones, colocando los resultados directamente en manos del estudiante.

El proceso de aprendizaje es enormemente dependiente del factor motivación, definiéndose ésta como el interés que tiene el estudiante por su propio aprendizaje o por las actividades que le conducen a él, y en la que podemos diferenciar entre motivación extrínseca y motivación intrínseca (Ryan, 2000). Hablamos de motivación extrínseca cuando una persona realiza una tarea para lograr un resultado (por ejemplo, un estudiante que estudia para un examen con el fin de obtener una buena calificación). Por el contrario, una persona que realiza una tarea por placer o interés está intrínsecamente motivado. La motivación intrínseca ha mostrado mejorar tanto el proceso como los resultados de aprendizaje. Por tanto, si el estudiante percibe una actividad como motivante, interesante y divertida, sus posibilidades de aprendizaje aumentan (Shute, 2013), siendo éste calificado como aprendizaje activo (Dias, 2017).

Respecto a los aspectos cognitivos implicados en el proceso y los resultados del aprendizaje, la neurociencia nos indica cuán numerosos factores influyen en ellos, comenzando por la percepción, la atención y la memoria, en base a las cuales puede alcanzarse la comprensión de conceptos, nociones e inferencias, siendo el fin último su integración aplicada en la resolución de problemas (Vermunt, 1996). Las actividades pedagógicas en las que los conceptos adquiridos deben aplicarse metacognitivamente, 
llevan implícita la auto-evaluación del nivel de comprensión de los contenidos y de la capacidad para relacionar conceptos (Schmitz, 2004). Consecuentemente, facilitan al estudiante la toma de conciencia y decisiones sobre sus capacidades, necesidades, y hábitos de estudio. Estos aspectos cognitivos son también recogidos por las teorías constructivistas (Vygotsky, 1980), basadas en que el conocimiento se construye a partir de nueva información que se asienta sobre los conceptos previamente existentes (Glaserfield, 1989). Por ello, si la actividad facilita la relación de conceptos nuevos con otros adquiridos previamente, será en teoría más eficaz como herramienta de aprendizaje.

Los diversos aspectos expuestos, auto-percibidos por los estudiantes respecto a sus recursos -seguridad en sus posibilidades, esfuerzo, motivación, comprensión y relación de conceptos-, se contemplan en las teorías del aprendizaje actuales, que proponen situar al estudiante en el centro del proceso de aprendizaje, como protagonista tanto del proceso como de los resultados alcanzados (Vermunt, 1996). En esta línea, se ha evidenciado cómo las metodologías de enseñanza basadas en la resolución de problemas (Problem Based Learning, denominado en adelante PBL por sus siglas en inglés) fomentan dichos factores intrínsecos del aprendizaje respecto a los formatos tradicionales basados en la clase magistral (Wood, 2003; Schmidt, 2011).

En ciencias de la salud, el trabajo consistente en la resolución de casos clínicos resulta una herramienta útil dentro de las metodologías PBL, requiriendo al estudiante una transferencia de los conocimientos teóricos adquiridos a los contextos prácticos donde tendrán que ser aplicados (Kolodner, 2003). A pesar de que estas prácticas de enseñanza llevan tiempo aplicándose en Fisioterapia, se encuentra poca evidencia sobre su efectividad en la formación en esta rama de la salud que nos ocupa. No obstante, la existente parece indicar estos mismos resultados positivos a los investigados en otras ciencias como Medicina y Enfermería, fomentando que los estudiantes apliquen conceptos importantes de su formación académica a situaciones aproximadas a los contextos clínicos reales (Solomon, 2005; Dyer, 2015), promoviendo la integración y relación conceptual, el proceso metacognitivo necesario para el razonamiento clínico, y la motivación intrínseca del alumno.

\subsection{Integración de las TIC y la lengua inglesa}

Por otra parte, el uso de las Tecnologías de la Información y Comunicación en docencia ha supuesto un gran avance, promoviendo el desarrollo de las competencias digitales en los estudiantes acorde a las necesidades tecnológicas y de autonomía que el mundo actual depara a los futuros profesionales (Greenhalgh, 2001; Nicklen 2016), y permitiendo también un mejor aprovechamiento del tiempo fuera del aula (Crawford, 2011).

Paralelamente, el uso del inglés como lengua franca se hace necesario en el contexto actual de globalización. La utilización de este idioma para la transmisión de contenidos y para la realización de actividades basadas en PBL como son los casos clínicos, requiere de un trabajo previo de los docentes en el diseño de la actividad, sirviéndose de metodologías como el Aprendizaje Integrado de Contenido y Lenguaje (Content and Language Integrated Learning, CLIL en adelante por sus siglas en inglés) (Coyle, 2010). El enfoque CLIL se integra dentro del caso clínico para mejorar la comprensión y accesibilidad de los contenidos presentados en inglés, a estudiantes cuya lengua materna no es ésta.

(cc)) BY-NC-ND 2017, Universitat Politècnica de València 
En el presente estudio, el equipo multidisciplinar encargado de diseñar la App y llevar a cabo la investigación, analiza las respuestas de los estudiantes frente a cuestiones sobre la utilidad global de la aplicación como herramienta de estudio, y sobre el papel de 'Labyrinth' en la facilitación de los factores intrínsecos del aprendizaje expuestos, considerando que ambos serán elementos clave en su proceso y resultados de aprendizaje.

En las siguientes secciones del artículo se describen los objetivos de la investigación, así como el diseño y características de la herramienta 'Labyrinth', del cuestionario de evaluación utilizado y del estudio piloto. Posteriormente se exponen y analizan los Resultados, que serán discutidos en el apartado final de Conclusiones, incluyendo posibles limitaciones y planteando nuevas líneas de investigación.

\section{OBJETIVOS}

La presente investigación se centró en explorar la percepción de los estudiantes respecto a la utilización de 'Labyrinth'como herramienta de aprendizaje para la resolución de casos clínicos. Específicamente se plantearon los siguientes objetivos:

- Evaluar la percepción de los estudiantes respecto a la utilidad global de 'Labyrinth' y de sus características específicas para alcanzar los objetivos de aprendizaje de la actividad.

- Cuantificar el grado en que los estudiantes vieron fomentados los factores intrínsecos del aprendizaje durante el uso de 'Labyrinth'.

- Observar si el grado de facilitación de dichos factores percibido por los estudiantes se relaciona con su valoración global de utilidad de 'Labyrinth' como herramienta de aprendizaje.

\section{DESARROLLO DE LA INNOVACIÓN}

\subsection{App 'Labyrinth'}

'Labyrinth', una aplicación basada en PBL (problem-based learning; Wood, 2003) accesible desde móviles, tablets y PCs, fue concebida para presentar casos clínicos sobre técnicas de electroterapia a los estudiantes de la asignatura "Procedimientos Generales de Fisioterapia I" (figura 1). La aplicación, íntegramente en inglés, sigue la metodología CLIL (Coyle 2011) para facilitar la comprensión de las instrucciones y los contenidos en dicha lengua.

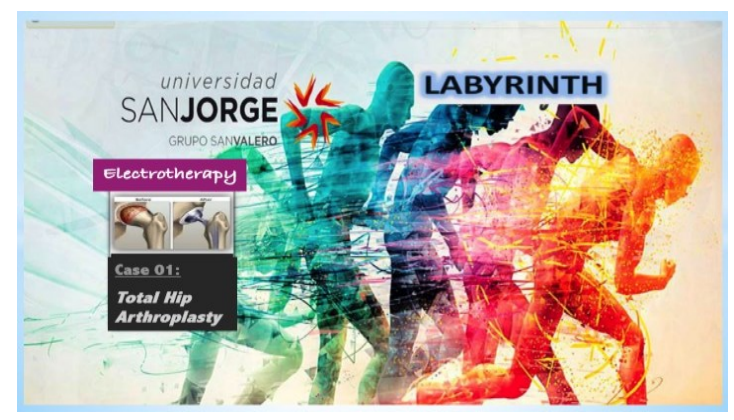

Figura 1. Pantalla inicial de Labyrinth'.

(cc)) EY-NC-ND 2017, Universitat Politècnica de València Congreso In-Red (2017) 
'Labyrinth' consiste en 6 niveles principales divididos a su vez en sub-niveles (figura 2).

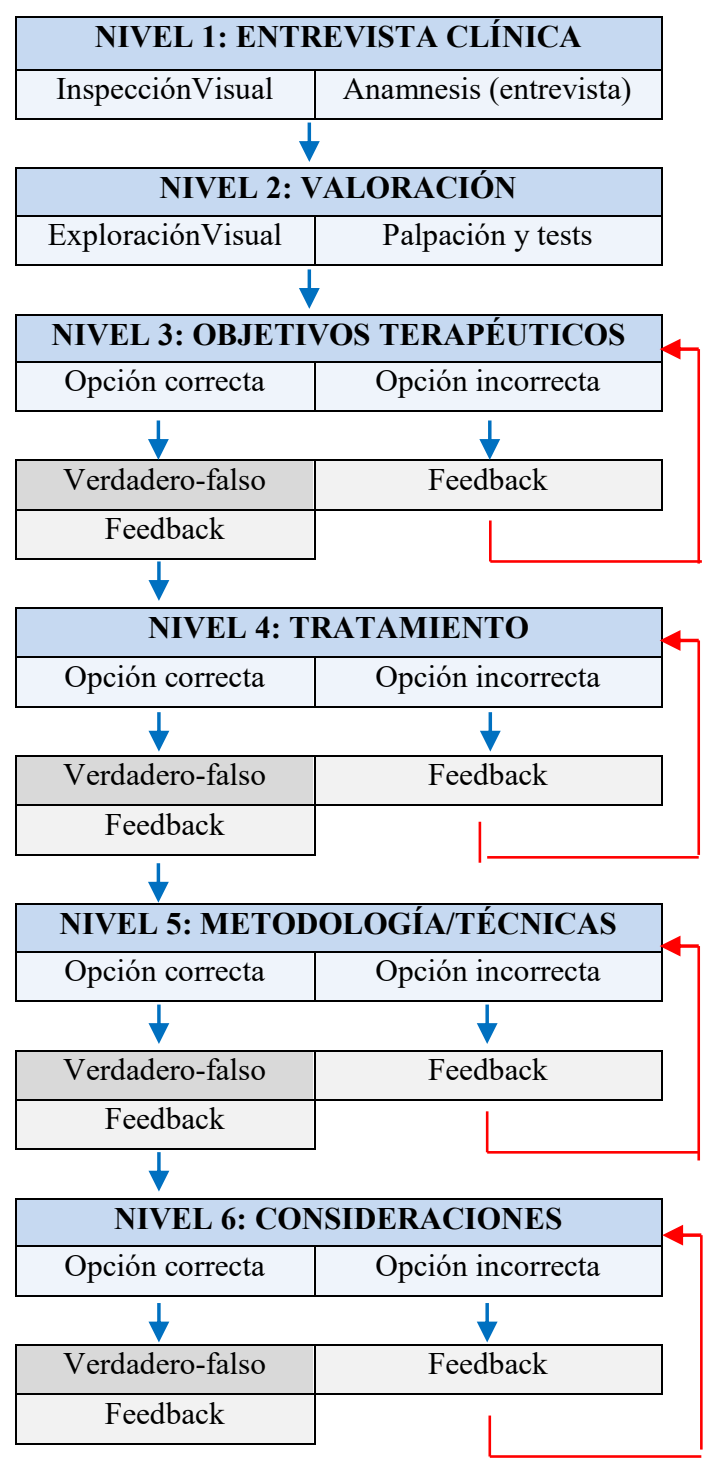

Figura 2. Niveles de la Aplicación 'Labyrinth'

La presentación del caso clínico comienza con la descripción y contextualización de un paciente. A continuación, a través de su uso en la interfaz para estudiantes, se requiere a los alumnos resolver el caso clínico presentado, debiendo para ello testar y relacionar sus conocimientos sobre la materia de electroterapia, tomando decisiones nivel tras nivel dentro del múltiple abanico de respuestas válidas que ofrece, y que dirigen hacia diferentes estrategias terapéuticas. 
En los niveles 1 y 2, los estudiantes deben tomar decisiones respecto a los factores más relevantes y específicos que deben conocer de la evaluación del paciente, en base a los cuales establecererán los objetivos de tratamiento.

En los niveles 3 a 6, utilizan la información conseguida anteriormente para proponer el abordaje terapéutico del problema clínico, identificando los objetivos de tratamiento y seleccionando las técnicas de electroterapia a aplicar, así como las precauciones y consideraciones a tener en cuenta en su patología.

En estos niveles, si el estudiante selecciona una opción incorrecta, recibe un feedback que le ayuda a comprender su equivocación y le redirige de nuevo a la selección de opciones. Por el contrario, si elige una opción correcta, debe completar un cuestionario "verdaderofalso" para confirmar el adecuado razonamiento clínico y descartar el azar en su acierto. Una vez este paso es completado con éxito, el estudiante puede acceder al siguiente nivel, y así consecutivamente hasta alcanzar la resolución del caso clínico.

El hecho de que existan varias opciones correctas en cada nivel, permite a los estudiantes avanzar en varias vías, de modo que el desconocimiento de una parte de la materia no impida la resolución del caso, sino fomente su búsqueda de alternativas en base a una nueva relación de conceptos.

La aplicación, a través de la interfaz para profesores, permite a los docentes facilitar información complementaria en cada nivel, en forma de documentos, links y material audiovisual, para apoyar el contraste de hipótesis en el razonamiento clínico del estudiante. Además permite acceder a los resultados de trabajo de los alumnos, así como a un foro en línea con los estudiantes.

\subsection{Encuestas de evaluación}

Se diseñó un cuestionario online anónimo con el objetivo de evaluar la percepción de los estudiantes respecto a la actividad, inmediatamente tras el uso de 'Labyrinth'.

En primer lugar los estudiantes registraron variables académicas (nota media del expediente académico, alumnos de primera o posteriores convocatorias y regularidad en su asistencia a las clases), así como datos demográficos (edad, género y nacionalidad), si bien en ningun momento revelaron su identidad.

El resto del cuestionario desarrollado consistió en escalas tipo Likert de 0 a 10 , exponiéndose afirmaciones en las que los estudiantes debían seleccionar un número conforme a su percepción respecto al enunciado, siendo 0 "estoy totalmente en desacuerdo/nunca" y 10 "estoy completamente de acuerdo/siempre". Dicho cuestionario se dividió en dos secciones que se exponen a continuación:

En la primera sección los estudiantes evaluaron el grado en el que la aplicación fomentó sus propios factores intrínsecos del aprendizaje, específicamente los siguientes:

1. Sentimiento de seguridad para poder superar la asignatura;

2. Motivación e interés por estudiar la asignatura;

3. Esfuerzo personal empleado en la actividad;

(cc)) EY-NC-ND 2017, Universitat Politècnica de València 
4. Comprensión de la materia;

5. Facilidad para relacionar los diferentes conceptos de la asignatura.

Así mismo se les solicitó asignar una puntuación a la valoración global de Labyrinth como herramienta de estudio y aplicación de conocimientos.

En la segunda sección los alumnos evaluaron de 0 a 10 el grado de utilidad de la App 'Labyrinth' y algunas de sus características, valorando específicamente:

1. Utilidad para resolver casos clínicos;

2. Utilidad para aplicar los conocimientos de la asignatura;

3. Utilidad para comprobar la integración de los contenidos de la asignatura;

4. Utilidad del foro en la resolución de dudas;

5. Dificultades debidas al idioma inglés;

6. Utilidad para preparar el examen;

7. Utilidad de la accesibilidad desde PC, tablet y móvil.

Se reservó un espacio al final del cuestionario donde se permitió a los estudiantes expresar cualquier tipo de observación o comentario.

\subsection{Estudio Piloto}

La intervención del presente estudio piloto tuvo lugar durante una clase habitual programada de 100 minutos de la asignatura "Procedimientos Generales de Fisioterapia I", que fue divididida en tres partes. Inicialmente el docente presentó el funcionamiento de Labyrinth. A continuación, durante 45 minutos y en grupos de 3-4 personas, los estudiantes trabajaron con Labyrinth en la resolución del caso clínico, siendo animados a utilizar un foro online habilitado para debatir y consultar entre ellos y con otros grupos. Los docentes también estuvieron disponibles para apoyar a los alumnos en caso de ser requerido.

Tras la finalización del tiempo de trabajo con Labyrinth, completaron el cuestionario online anónimo durante 20 minutos.

\section{RESULTADOS}

\subsection{Análisis Estadístico}

Para categorizar las características basales de la muestra, las variables se describen como número (porcentaje) y media (desviación estándar). Las variables cuantitativas se sometieron al test Kolmogorov-Smirnov para determinar su distribución y utilizar las pruebas estadísticas correctas acorde con los resultados. La correlación entre las variables se determinó mediante el cálculo del coeficiente de correlación de Pearson o de Spearman según la normalidad o no de dichas variables.

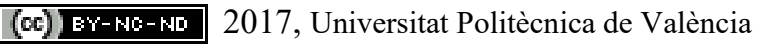




\subsection{Resultados de correlaciones}

La correlación entre la valoración global de la aplicación y cada uno de los ítems que evaluaban el nivel de satisfacción de los estudiantes con sus factores intrínsecos del aprendizaje, fue elevada: $\mathrm{r}>0.83$ y $\mathrm{p}<0.001$ en todos los casos (figuras 1 a 5).

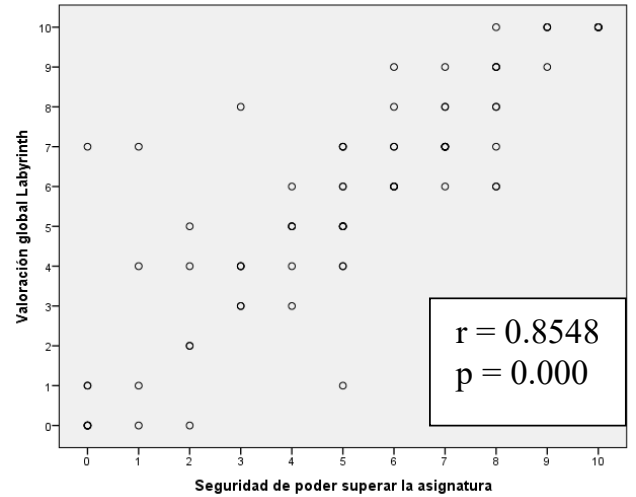

Figura 1. Gráfico de dispersión entre la valoración global de 'Labyrinth' y la seguridad de poder superar la asignatura. $p$ : valor-p; $r$ : coeficiente de correlación de Pearson.

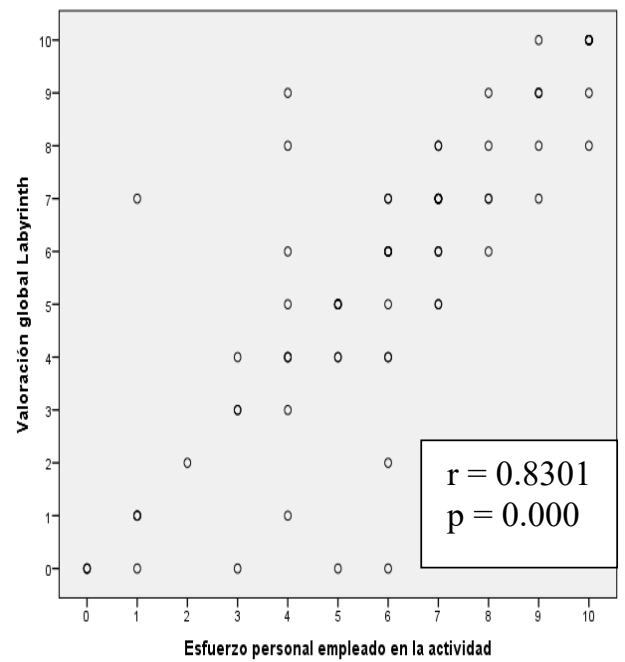

Figura 3. Gráfico de dispersión entre la valoración global de 'Labyrinth' y la percepción de esfuerzo personal empleado. $p$ : valor-p; $r$ : coeficiente de correlación de Pearson.

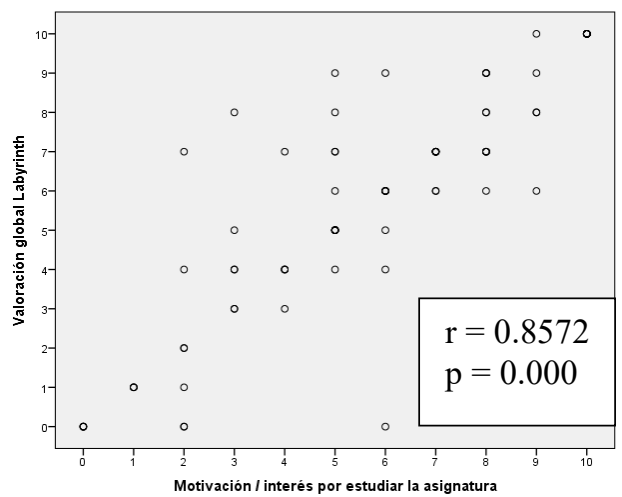

Figura 2. Gráfico de dispersión entre la valoración global de 'Labyrinth' y la motivación-interés por estudiar la asignatura. $p$ : valor-p; $r$ : coeficiente de correlación de Pearson.

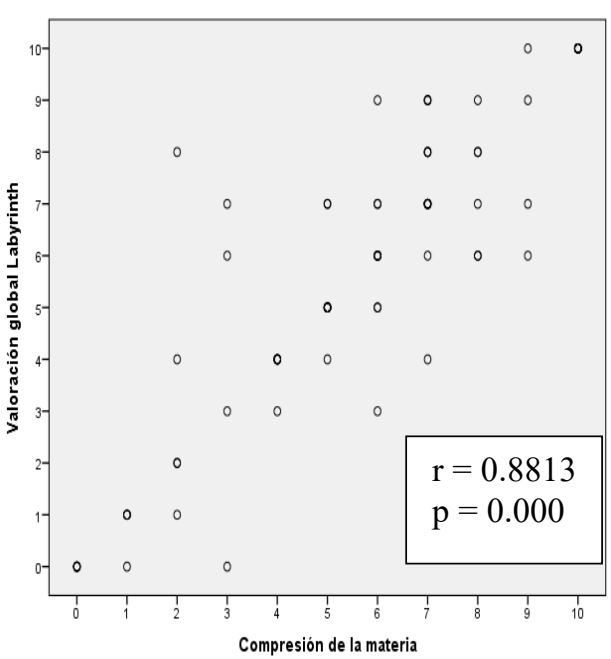

Figura 4. Gráfico de dispersión entre la valoración global de 'Labyrinth' y la ayuda que supone para comprender la materia. $p$ : valor-p; $r$ : coeficiente de correlación de Pearson. 


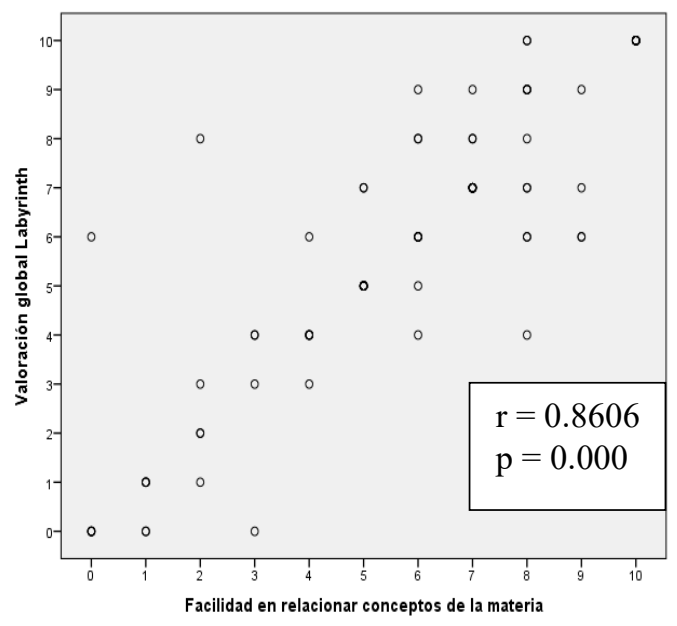

Figura 5. Gráfico de dispersión entre la valoración global de 'Labyrinth' y la facilidad en relacionar conceptos de la materia. $p$ : valor-p; $r$ : coeficiente de correlación de Pearson.

La relación entre la percepción de los usuarios sobre el grado en que 'Labyrinth'fomentaba estos factores intrínsecos claves para el aprendizaje y otras variables analizadas -donde se valoraba la aplicación como herramienta de ayuda para el aprendizaje (resolución de casos clínicos, aplicación de los conocimientos de la asignatura, comprobar el aprendizaje de los conceptos de la asignatura), así como la utilidad de las características de la aplicación (resolución de dudas a través de un foro, uso del inglés como lenguaje vehicular, poder trabajar con la aplicación fuera de clase, acceso a través de PC, Tablet y móvil), también tuvieron valores de correlación aceptables y significativos, aunque menores que los comentados previamente: $\mathrm{r}>0.64$ y $\mathrm{p}<0.001$; con la excepción del inglés como lenguaje vehicular de la aplicación, donde no se encontró ningún tipo de correlación.

Las Tablas 2 y 3 muestran los resultados expuestos de correlaciones entre cada uno de los factores intrínsecos del aprendizaje percibidos por los estudiantes, y su valoración de la utilidad de Labyrinth, global y específica.

Tabla 2. Resultados de correlaciones respecto a la valoración global de utilidad de 'Labyrinth'.

\begin{tabular}{|l|c|c|c|c|c|}
\hline \multirow{2}{*}{$n=79$} & \multicolumn{5}{|c|}{ FACTORES INTRÍNSECOS DEL APRENDIZAJE } \\
\hline \multirow{2}{*}{$\begin{array}{c}\text { UTILIDAD DE } \\
\text { 'LABYRINTH': }\end{array}$} & $\begin{array}{c}\text { Seguridad } \\
\text { de poder } \\
\text { superar la } \\
\text { asignatura }\end{array}$ & $\begin{array}{c}\text { Motiva- } \\
\text { ción por } \\
\text { estudiar la } \\
\text { asignatura }\end{array}$ & $\begin{array}{c}\text { Esfuerzo } \\
\text { personal } \\
\text { empleado }\end{array}$ & $\begin{array}{c}\text { Compren- } \\
\text { sión de la } \\
\text { materia }\end{array}$ & $\begin{array}{c}\text { Facilidad } \\
\text { para } \\
\text { relacionar } \\
\text { conceptos }\end{array}$ \\
\hline Valoración global & $r=0.8548^{*}$ & $r=0.857^{*}$ & $r=0.8813^{*}$ & $r=0.8301^{*}$ & $r=0.8606^{*}$ \\
& $p=0,0000$ & $p=0,0000$ & $p=0,0000$ & $p=0,0000$ & $p=0,0000$ \\
\hline
\end{tabular}


Tabla 3. Resultados de correlaciones respecto a la valoración de la utilidad específica de 'Labyrinth'.

\begin{tabular}{|c|c|c|c|c|c|}
\hline$n=79$ & \multicolumn{5}{|c|}{ FACTORES INTRÍNSECOS DEL APRENDIZAJE } \\
\hline $\begin{array}{l}\text { UTILIDAD DE } \\
\text { 'LABYRINTH': }\end{array}$ & $\begin{array}{c}\text { Seguridad } \\
\text { de poder } \\
\text { superar la } \\
\text { asignatura }\end{array}$ & $\begin{array}{c}\text { Motiva- } \\
\text { ción por } \\
\text { estudiar la } \\
\text { asignatura }\end{array}$ & $\begin{array}{r}\text { Esfuerzo } \\
\text { personal } \\
\text { empleado }\end{array}$ & $\begin{array}{l}\text { Compren- } \\
\text { sión de la } \\
\text { materia }\end{array}$ & $\begin{array}{l}\text { Facilidad } \\
\text { para } \\
\text { relacionar } \\
\text { conceptos }\end{array}$ \\
\hline $\begin{array}{l}\text { Herramienta útil para } \\
\text { resolver casos clínicos }\end{array}$ & $\begin{array}{l}r=0.7076 \\
p=0,0000\end{array}$ & $\begin{array}{l}r=0.7179 \\
p=0,0000\end{array}$ & $\begin{array}{l}r=0.7413 \\
p=0,0000\end{array}$ & $\begin{array}{l}r=0.7707 \\
p=0,0000\end{array}$ & $\begin{array}{l}r=0.6776 \\
p=0,0000\end{array}$ \\
\hline $\begin{array}{l}\text { Herramienta útil para } \\
\text { aplicar conocimientos }\end{array}$ & $\begin{array}{l}r=0.7043 \\
p=0,0000\end{array}$ & $\begin{array}{l}r=0.7169 \\
p=0,0000\end{array}$ & $\begin{array}{l}r=0.6929 \\
p=0,0000\end{array}$ & $\begin{array}{l}r=0.7640 \\
p=0,0000\end{array}$ & $\begin{array}{l}r=0.6915 \\
p=0,0000\end{array}$ \\
\hline $\begin{array}{l}\text { Me permite compro- } \\
\text { bar si he aprendido } \\
\text { los conceptos }\end{array}$ & $\begin{array}{l}r=0.6627 \\
p=0,0000\end{array}$ & $\begin{array}{l}r=0.6976 \\
p=0,0000\end{array}$ & $\begin{array}{l}r=0.6960 \\
p=0,0000\end{array}$ & $\begin{array}{l}r=0.7302 \\
p=0,0000\end{array}$ & $\begin{array}{l}r=0.6791 \\
p=0,0000\end{array}$ \\
\hline $\begin{array}{l}\text { La resolución de } \\
\text { dudas a través del } \\
\text { foro ha sido útil }\end{array}$ & $\begin{array}{l}r=0.6726 \\
p=0,0000\end{array}$ & $\begin{array}{l}r=0.7035 \\
p=0,0000\end{array}$ & $\begin{array}{l}r=0.5947 \\
p=0,0000\end{array}$ & $\begin{array}{l}r=0.7473 \\
p=0,0000\end{array}$ & $\begin{array}{l}r=0.6506 \\
p=0,0000\end{array}$ \\
\hline $\begin{array}{l}\text { El hecho de que esté } \\
\text { en inglés me supone } \\
\text { un impedimento }\end{array}$ & $\begin{array}{l}r=0.1386 \\
p=0,0000\end{array}$ & $\begin{array}{l}r=0.1723 \\
p=0,0000\end{array}$ & $\begin{array}{l}r=0.1676 \\
p=0,0000\end{array}$ & $\begin{array}{l}r=0.2111 \\
p=0,0000\end{array}$ & $\begin{array}{l}r=0.1692 \\
p=0,0000\end{array}$ \\
\hline $\begin{array}{l}\text { Me gustaría trabajar } \\
\text { con la App para } \\
\text { preparar examen }\end{array}$ & $\begin{array}{l}r=0.6855 \\
p=0,0000\end{array}$ & $\begin{array}{l}r=0.7416 \\
p=0,0000\end{array}$ & $\begin{array}{l}r=0.7000 \\
p=0,0000\end{array}$ & $\begin{array}{l}r=0.6840 \\
p=0,0000\end{array}$ & $\begin{array}{l}r=0.6419 \\
p=0,0000\end{array}$ \\
\hline $\begin{array}{l}\text { Es útil que esté } \\
\text { disponible en móvil, } \\
\text { tablet y PC }\end{array}$ & $\begin{array}{l}r=0.7273 \\
p=0,0000\end{array}$ & $\begin{array}{l}r=0.7225 \\
p=0,0000\end{array}$ & $\begin{array}{l}r=0.6300 \\
p=0,0000\end{array}$ & $\begin{array}{l}r=0.7430 \\
p=0,0000\end{array}$ & $\begin{array}{l}r=0.6833 \\
p=0,0000\end{array}$ \\
\hline
\end{tabular}

\section{CONCLUSIONES}

Los resultados de la encuesta muestran que la valoración de la utilidad de la aplicación tiene una relación directamente proporcional con los factores intrinsecos que su uso fomenta (seguridad, motivación, esfuerzo, comprensión y capacidad de relación de conceptos), y que se consideran clave para el aprendizaje.

Por tanto se cumplen nuestros objetivos tenidos en cuenta en el diseño de Labyrinth, pudiendo concluir que sus características -posibilidad de diferentes respuestas válidas, permitiendo diferentes procesos de razonamiento potencialmente correctos y por tanto más adaptados a la realidad de la práctica clínica; fomento de la auto-reflexión tras cada respuesta testando su veracidad mediante preguntas específicas sobre su selección, y accediendo a un feedback posterior; acceso a un foro en línea y a material específico de 
consulta y profundización de conocimientos a través de la propia aplicación; posibilidad de varios intentos, disminuyendo la frustración en el proceso de llegar a una solución final; así como su accesibilidad desde el teléfono móvil adaptadándose a la ubicuidad del actual mundo-, se relacionan con mayor satisfacción percibida por los usuarios respecto a sus propios recursos atitudinales y cognitivos.

Así mismo, el diseño del cuestionario de evaluación de Labyrinth persiguió el objetivo de aumentar la conciencia de los estudiantes sobre cómo sus propias percepciones de seguridad, motivación, esfuerzo y capacidades cognitivas, influyen en su consecución de los objetivos de aprendizaje. Con este fin, la elaboración de la encuesta se basó en el cuestionario SALG (student assessment of learning gains; Seymour, 2000), cuyos enunciados están destinados a fomentar la reflexión de los estudiantes sobre qué, cómo y cuánto han aprendido a través de diferentes actividades y enfoques pedagógicos. El citado cuestionario se fundamenta en la asunción de que los estudiantes, aunque quizá no capacitados para evaluar el desempeño del profesor, son capaces de estimar de manera realista el grado en que ciertos aspectos metodológicos empleados durante diferentes actividades pedagógicas fomenta su aprendizaje. Los resultados obtenidos mediante el uso de esta herramienta de evaluación adaptada del cuestionario SALG, en base a la percepción de los estudiantes, nos han permitido identificar los elementos que contribuyen y asisten el aprendizaje, y aquellos otros que podrían ser mejorados en aspectos concretos.

En este sentido, el hecho de que los contenidos de la aplicación estén desarrollados íntegramente en inglés podría suponer un impedimento para fomentar los factores intrínsecos del aprendizaje, motivo por el cual se invirtió trabajo en su facilitación mediante la metodología CLIL. Finalmente podemos concluir que los estudiantes no perciben dificultades añadidas por esta causa, ya que el uso de inglés como lenguaje vehicular fue el único ítem que no se correlacionó positiva ni negativamente con dichos factores.

El diseño observacional de este estudio piloto nos ha permitido detectar y determinar la relación positiva existente entre la percepción de los usuarios sobre cómo Labyritnh promueve estos factores intrinsecos del aprendizaje, y la valoración global que otorgan a la aplicación y a sus características. Estos hallazgos abren nuevas líneas de investigación sobre la importancia de estos factores en el proceso de enseñanza-aprendizaje, no habiendo sido explorados en profundidad en el campo de la formación de profesionales de la salud, y pocas veces tenidos en cuenta por la evidencia científica.

La reflexión y toma de conciencia sobre estos factores es imprescindible para la optimización de las estrategias docentes y de los hábitos de estudio.

\section{REFERENCIAS BIBLIOGRÁFICAS}

BLACKWELL, L., TRZESNIEWSKI, K., y DWECK, C.S. (2007). "Implicit theories of intelligence predict achievement across an adolescent transition: A longitudinal study and intervention". Child Development, 78: 246-263.

COYLE, D., HOOD, P., MARSH, D. (2010). CLIL: Content and Language Integrated Learning: Cambridge University Press.

(c)) BY-NC-ND 2017, Universitat Politècnica de València 
CRAWFORD, T.R. (2011). "Using problem-based learning in web-based components of nurse education”. Nurse Educ Pract, 11 (2): 124-130.

DIAS, J. (2017). "Teaching operations research to undergraduate management students: The role of gamification”. The International Journal of Management Education, 15: 98-111.

DWECK, C.S. (2007). Mindset: The new psychology of success. New York: Ballantine Books.

DYER, J.O., HUDON, A., MONTPETIT-TOURANGEAU, K., CHARLIN, B., et al. (2015). "Example-based learning: comparing the effects of additionally providing three different integrative learning activities on physiotherapy intervention knowledge". BMC Med Educ, 15: 37-49.

GLASERSFELD, E. (1989). Cognition, construction of knowledge, and teaching. Synthese, 80 (1): $121-140$

GOOD, C., ARONSON, J., e INZLICHT, M. (2003). "Improving adolescents' standardized test performance: An intervention to reduce the effects of stereotype threat". Applied Developmental Psychology, 24: 645-662.

GREENHALGH, T. (2001). "Computer assisted learning in undergraduate medical education". British Medical Journal, 322 (7277), 40-44.

KOLODNER, J.L., CAMP, P.J., CRISMOND, D., FASSE, B, et al (2003). "Problem-Based Learning Meets Case-Based Reasoning in the Middle-School Science Classroom: Putting Learning by Design(tm) Into Practice". Journal of the Learning Sciences, 12 (4): 495-547.

NICKLEN, P., KEATING, J.L., MALONEY, S. (2016). "Exploring Student Preconceptions of Readiness for Remote-Online Case-Based Learning: A Case Study", JMIR Medical Education, 2 (1): 1-9.

PAJARES, F. (1996b) "Assessing self-efficacy beliefs and mathematical problem solving of gifted students". Contemporary Educational Psychology, 21: 325-344.

RYAN, R.M. y DECI, E.L. (2000). "Intrinsic and Extrinsic Motivations: Classic Definitions and New Directions”. Contemporary Educational Psychology, 25: 54-67.

SCHMIDT, H.G., ROTGANS, J.I., YEW, E.H. (2011). "The process of problem-based learning: what works and why". Med Educ, 45 (8): 792-806.

SCHMITZ, T.W., KAWAHARA-BACCUS, T.N. y JOHNSON, S.C. (2004). "Metacognitive evaluation, self-relevance, and the right prefrontal cortex". NeuroImage, 22: 941-947

SEYMOUR, E., WIESE, D., HUNTER, A., DAFFINRUD, S.M. (2000). Creating a better mousetrap: on-line student assessment of their learning gains; National Meeting of the American Chemical Society; San Francisco, CA.

SHUTE, V.J. Y VENTURA, M. (2013). Stealth assessment: Measuring and supporting learning in games. Cambridge, MA: Massachusetts Institute of Technology Press Books.

SOLOMON, P. (2005), "Problem-based Learning: A review of current issues relevant to physiotherapy education". Physiotherapy Theory and Practice, 21 (1): 37-49.

VERMUNT, J.D. (1996). "Metacognitive, cognitive and affective aspects of learning styles and strategies: A phenomenographic analysis". Higher Education, 31: 25-50.

VYGOTSKY, L.S. (1980). Mind in society: The development of higher psychological processes. Harvard university press.

WOOD, D.F. (2003). "Problem based learning". BMJ, 326 (7384): 328-330. 